hep-th/0301109

CERN-TH/2003-005

SISSA 5/2003/EP

\title{
Semiclassical decay of strings with maximum angular momentum
}

\author{
Roberto Iengo $^{a}$ and Jorge G. Russo ${ }^{b, c}$ \\ a International School for Advanced Studies (SISSA), Via Beirut 2-4, \\ I-34013 Trieste, Italy \\ INFN, Sezione di Trieste \\ b Theory Division, CERN, CH-1211 Geneva, Switzerland \\ ${ }^{c}$ Departamento de Física, Universidad de Buenos Aires and Conicet, \\ Ciudad Universitaria, Pab. I, 1428 Buenos Aires, Argentina
}

\begin{abstract}
We study the classical breaking of a highly excited (closed or open) string state on the leading Regge trajectory, represented by a rotating soliton solution, and we find the resulting solutions for the outgoing two pieces, describing two specific excited string states. This classical picture reproduces very accurately the precise analytical relation of the masses $M_{1}$ and $M_{2}$ of the decay products found in a previous quantum computation. The decay rate is naturally described in terms of a semiclassical formula. We also point out some interesting features of the evolution after the splitting process.
\end{abstract}




\section{Contents}

\section{Introduction}

\section{Splitting of a rotating string}

2.1 Fourier analysis

2.2 Closed formulas for the outgoing string solutions

\section{Comparison with the superstring quantum calculation}

\section{Decay rate and description of the motion - Figures for the string splitting and evolution}

\section{Introduction}

The study of the decay properties of massive string states, and, in particular, the attempt to determine their lifetime, was initiated long ago, with various kinds of results [1, 2, 3, 4, 5, 6, 17, 8, 9, 10, 11, sometimes finding a long lifetime (see e.g. [1, 6]). The main difficulty is represented by the high degeneracy of the decay products, even for the splitting into two bodies, which can be viewed as the first step of the decay process.

Here we present a simple classical computation, which is applicable for large quantum numbers, describing the splitting into two pieces of a very massive closed string in the state of maximal, and thus very large, angular momentum (a study of classical splitting of a different circular pulsating string is in [12]). This computation will prescribe a definite relation between the masses of the decay products and will also give information about their angular momentum. Moreover, the semiclassical argument indicates that the lifetime for the splitting of such highly excited states will grow proportional to its mass.

We will also compare the results of this semiclassical picture with the detailed quantum computation that we recently performed on the decay of a massive state with maximal angular momentum in closed superstring theory [1]. The quantum computation was based on evaluating the imaginary part of the one-loop self-energy of that state. The self-energy is expressed as an integral of a certain combination of theta functions, and we developed a very 
efficient algorithm to derive the contribution of the intermediate states of definite mass, which included the sum over their degeneracies.

The comparison will show a surprisingly good agreement between the semiclasssical and quantum calculation, despite the fact they involve completely different computations.

\section{Splitting of a rotating string}

The rotating closed string solution is given by:

$$
X=L \cos (2 \sigma) \cos (2 \tau), \quad Y=L \cos (2 \sigma) \sin (2 \tau), \quad X^{0}=2 L \tau,
$$

where $\sigma \in[0, \pi)$. This represents a spinning folded closed string with maximum angular momentum. The coefficient $2 L$ in $X^{0}$ is fixed by the constraint $\dot{X} \cdot \dot{X}+X^{\prime} \cdot X^{\prime}=0$ (the other constraint $\dot{X} \cdot X^{\prime}=0$ is also satisfied). The string has energy and angular momentum given by

$$
E=M=\frac{L}{\alpha^{\prime}}, \quad J=\frac{L^{2}}{2 \alpha^{\prime}},
$$

so that one has the usual Regge relation $\alpha^{\prime} M^{2}=2 J$.

The closed string (2.1) contains two segments, the "upper" segment described by $0<\sigma<\frac{\pi}{2}$ and the "lower" segment described by $\frac{\pi}{2}<\sigma<\pi$. The full length of the string is $4 L$. We assume that at $\tau=0$ the string splits into two pieces of lengths $4 L_{\mathrm{I}}$ and $4 L_{\mathrm{II}}, X^{\mu}(\sigma) \rightarrow\left\{X_{\mathrm{I}}^{\mu}(\sigma), X_{\mathrm{II}}^{\mu}(\sigma)\right\}$, with $L_{\mathrm{I}}+L_{\mathrm{II}}=L$. The initial conditions for the strings I and II are given by the string (2.1) at $\tau=0$. The splitting occurs at $\sigma=\frac{a \pi}{2}$ for the upper segment, with $a$ defined as

$$
\cos a \pi \equiv-\frac{L_{\mathrm{I}}-L_{\mathrm{II}}}{L}, \quad 0<a<1
$$

For the lower segment, the splitting is at $\sigma=\pi-\frac{a \pi}{2}$. Thus the initial conditions are:

$$
X_{\mathrm{I}}^{\mu}(\sigma, 0)=X^{\mu}(\sigma, 0), \quad \dot{X}_{\mathrm{I}}^{\mu}(\sigma, 0)=\dot{X}^{\mu}(\sigma, 0),
$$

for $0<\sigma<\frac{a \pi}{2}$ (upper segment) and for $\pi>\sigma>\pi-\frac{a \pi}{2}$ (lower segment), while for the string II we have

$$
X_{\mathrm{II}}^{\mu}(\sigma, 0)=X^{\mu}(\sigma, 0), \quad \dot{X}_{\mathrm{II}}^{\mu}(\sigma, 0)=\dot{X}^{\mu}(\sigma, 0),
$$


for $\frac{a \pi}{2}<\sigma<\frac{\pi}{2}$ (upper segment) and for $\frac{\pi}{2}<\sigma<\pi-\frac{a \pi}{2}$ (lower segment). These boundary conditions uniquely determine the solution describing the two closed string final states.

We shall determine the solution describing the two outgoing solutions in two different ways:

a) By explicitly finding the Fourier modes. This will show, in particular, that the outgoing strings are in a highly excited state which is not of maximum angular momentum.

b) By a direct matching with the original solution and imposing the new periodicity. By construction, the resulting solutions coincide with the explicit calculation of a), and thus this method provides a simple closed analytic formula for the resummation of the Fourier expansion. This form of the solution also exhibits the nature of the motion of the outgoing strings I and II.

\subsection{Fourier analysis}

Let us consider the string I. To find the explicit solution, we start with the general solution to the string equations for the closed string. The condition (2.4) can be equivalently imposed in the interval $-\frac{a \pi}{2}>\sigma>\frac{a \pi}{2}$, since the original solution is periodic in $\sigma$ with period $\pi$. The most general closed string solution satisfying the periodicity condition $X_{\mathrm{I}}^{\mu}(\sigma+\pi a)=X_{\mathrm{I}}^{\mu}(\sigma)$ is given by

$$
\begin{aligned}
& X_{\mathrm{I}}(\sigma, \tau)=x_{0 \mathrm{I}}+2 \alpha^{\prime} p_{\mathrm{I}}^{x} \frac{\tau}{a}+i \sum_{n \neq 0}\left(x_{n} e^{-2 i \frac{n}{a}(\tau-\sigma)}+\tilde{x}_{n} e^{-2 i \frac{n}{a}(\tau+\sigma)}\right), \\
& Y_{\mathrm{I}}(\sigma, \tau)=y_{0 \mathrm{I}}+2 \alpha^{\prime} p_{\mathrm{I}}^{y} \frac{\tau}{a}+i \sum_{n \neq 0}\left(y_{n} e^{-2 i \frac{n}{a}(\tau-\sigma)}+\tilde{y}_{n} e^{-2 i \frac{n}{a}(\tau+\sigma)}\right) .
\end{aligned}
$$

Note that the constraints

$$
\dot{X} \cdot X^{\prime}=0, \quad \dot{X} \cdot \dot{X}+X^{\prime} \cdot X^{\prime}=0,
$$

are satisfied automatically once we impose the initial conditions. Indeed, they are satisfied at $\tau=0$, because there the solutions $X_{I}^{\mu}, X_{I I}^{\mu}$ and their first derivatives coincide with the original solution $X^{\mu}$, which already satisfies the constraints. Since they are a constant of motion, then they are satisfied for all $\tau$ (using the fact that $T_{++}=T_{++}\left(\sigma^{+}\right), T_{--}=T_{--}\left(\sigma^{-}\right)$, one sees 
that the condition $T_{++}=T_{--}=0$ at $\tau=0$ implies that they vanish for any $\tau)$.

Let us first determine the conserved quantum numbers, energy, linear momentum and angular momentum of each string. Since they are conserved quantities, they can be found at $\tau=0$, where the solution is given by eq. (2.4), (2.5). The energy and linear momentum components are given by

$$
\begin{aligned}
E_{\mathrm{I}} & =\frac{2}{2 \pi \alpha^{\prime}} \int_{0}^{\frac{\pi a}{2}} d \sigma \dot{X}_{\mathrm{I}}^{0}=\frac{L a}{\alpha^{\prime}} \\
p_{\mathrm{I}}^{x} & =0 \\
p_{\mathrm{I}}^{y} & =\frac{2}{2 \pi \alpha^{\prime}} \int_{0}^{\frac{\pi a}{2}} d \sigma \dot{Y}_{\mathrm{I}}=\frac{4 L}{2 \pi \alpha^{\prime}} \int_{0}^{\frac{\pi a}{2}} d \sigma \cos (2 \sigma)=\frac{L \sin (\pi a)}{\pi \alpha^{\prime}} .
\end{aligned}
$$

There is an extra factor of two in the above expressions, which takes into account that there are two segments of string giving the same contribution. The angular momentum is

$$
J_{\mathrm{I}}=\frac{2}{2 \pi \alpha^{\prime}} \int_{0}^{\frac{\pi a}{2}}\left(X_{\mathrm{I}} \dot{Y}_{\mathrm{I}}-\dot{X}_{\mathrm{I}} Y_{\mathrm{I}}\right)=\frac{L^{2} a}{2 \alpha^{\prime}}\left(1+\frac{\sin (2 \pi a)}{2 \pi a}\right) .
$$

This has an orbital component $l_{\mathrm{I}}$ and spin component $S_{\mathrm{I}}$. Since $p_{\mathrm{I}}^{x}=0$, the orbital component is just $l_{\mathrm{I}}=x_{0 \mathrm{I}} p_{\mathrm{I}}^{y}$, where $x_{0 \mathrm{I}}$ is the center of mass coordinate of the string I,

$$
x_{0 \mathrm{I}}=\frac{2}{\pi a} \int_{0}^{\frac{\pi a}{2}} d \sigma X_{\mathrm{I}}=\frac{L \sin (\pi a)}{\pi a}=\frac{\alpha^{\prime}}{a} p_{\mathrm{I}}^{y} .
$$

Therefore $J_{\mathrm{I}}=l_{\mathrm{I}}+S_{\mathrm{I}}$ with

$$
l_{\mathrm{I}}=\frac{L^{2} a}{\alpha^{\prime}} \frac{\sin ^{2}(\pi a)}{(\pi a)^{2}}, \quad S_{\mathrm{I}}=\frac{L^{2} a}{2 \alpha^{\prime}}\left(1-\frac{2 \sin ^{2}(\pi a)}{(\pi a)^{2}}+\frac{\sin (2 \pi a)}{2 \pi a}\right) .
$$

The mass of the string I is thus given by

$$
M_{\mathrm{I}}^{2}=E_{\mathrm{I}}^{2}-p_{\mathrm{I}}^{2}=\frac{L^{2}}{\alpha^{2}}\left(a^{2}-\frac{\sin ^{2}(\pi a)}{\pi^{2}}\right) .
$$

Let us now determine the oscillator modes. From the conditions $\dot{X}_{\mathrm{I}}(\sigma, 0)=$ $\dot{X}(\sigma, 0)=0, Y_{\mathrm{I}}(\sigma, 0)=Y(\sigma, 0)=0$, it follows that

$$
x_{n}=\tilde{x}_{-n}, \quad y_{n}=-\tilde{y}_{-n} .
$$


To find $x_{n}, y_{n}$, we multiply the two remaining boundary conditions $X_{\mathrm{I}}(\sigma, 0)=$ $X(\sigma, 0), \dot{Y}_{\mathrm{I}}(\sigma, 0)=\dot{Y}(\sigma, 0)=0$ by $e^{-2 i \frac{n}{a} \sigma}$ and perform the integral over $\sigma$ from $-\frac{a \pi}{2}$ to $\frac{a \pi}{2}$, using the expansions (2.6), (2.7) and the solution (2.1). We find

$$
\begin{gathered}
X_{\mathrm{I}}=\frac{L \sin (\pi a)}{\pi a}\left(1+2 \sum_{n=1}^{\infty} \frac{(-1)^{n}}{1-\frac{n^{2}}{a^{2}}} \cos \left(\frac{2 n \tau}{a}\right) \cos \left(\frac{2 n \sigma}{a}\right)\right), \\
Y_{\mathrm{I}}=\frac{L \sin (\pi a)}{\pi a}\left(2 \tau+2 a \sum_{n=1}^{\infty} \frac{(-1)^{n}}{n\left(1-\frac{n^{2}}{a^{2}}\right)} \sin \left(\frac{2 n \tau}{a}\right) \cos \left(\frac{2 n \sigma}{a}\right)\right),
\end{gathered}
$$

where $-\frac{\pi a}{2}<\sigma<\frac{\pi a}{2}$ (note that the factor $(-1)^{n}$ can be removed byshifting $\sigma$ so that $0<\sigma<\pi a$ ). Finally, we have $X_{\mathrm{I}}^{0}=2 L \tau$.

Let us now consider the string II. The solution is readily found by noting that the Fourier analysis become the same in terms of $a^{\prime}=1-a$ and $\sigma^{\prime}=$ $\sigma-\frac{\pi}{2}$. We have to take into account that the shift in $\sigma$ produces a change of sign in the solution (2.1). We get

$$
\begin{gathered}
X_{\mathrm{II}}=-\frac{L \sin (\pi a)}{\pi(1-a)}\left(1+2 \sum_{n=1}^{\infty} \frac{(-1)^{n}}{1-\frac{n^{2}}{(1-a)^{2}}} \cos \left(\frac{2 n \tau}{(1-a)}\right) \cos \left(\frac{2 n \sigma}{(1-a)}\right)\right) \\
Y_{\mathrm{II}}=-\frac{L \sin (\pi a)}{\pi(1-a)}\left(2 \tau+2(1-a) \sum_{n=1}^{\infty} \frac{(-1)^{n}}{n\left(1-\frac{n^{2}}{(1-a)^{2}}\right)} \sin \left(\frac{2 n \tau}{(1-a)}\right) \cos \left(\frac{2 n \sigma}{(1-a)}\right)\right)
\end{gathered}
$$

with $-\frac{\pi(1-a)}{2}<\sigma<\frac{\pi(1-a)}{2}$, and $X_{\mathrm{II}}^{0}=2 L \tau$.

The conserved quantities for the string II are

$$
\begin{gathered}
E_{\mathrm{II}}=\frac{L(1-a)}{\alpha^{\prime}}, \quad p_{\mathrm{II}}^{y}=-\frac{L \sin (\pi a)}{\pi \alpha^{\prime}}, \\
J_{\mathrm{II}}=l_{\mathrm{II}}+S_{\mathrm{II}}, \quad l_{\mathrm{II}}=\frac{L^{2}(1-a)}{\alpha^{\prime}} \frac{\sin ^{2}(\pi a)}{(\pi(1-a))^{2}}, \\
S_{\mathrm{II}}=\frac{L^{2}(1-a)}{2 \alpha^{\prime}}\left(1-\frac{2 \sin ^{2}(\pi a)}{(\pi(1-a))^{2}}-\frac{\sin (2 \pi a)}{2 \pi(1-a)}\right), \\
M_{\mathrm{II}}^{2}=E_{\mathrm{II}}^{2}-p_{\mathrm{II}}^{2}=\frac{L^{2}}{\alpha^{\prime 2}}\left((1-a)^{2}-\frac{\sin ^{2}(\pi a)}{\pi^{2}}\right) .
\end{gathered}
$$

One easily checks that energy, linear momentum and angular momentum are conserved in the process of splitting,

$$
E_{\mathrm{I}}+E_{\mathrm{II}}=\frac{L}{\alpha^{\prime}}=E, \quad p_{\mathrm{I}}^{y}+p_{\mathrm{II}}^{y}=0, \quad J_{\mathrm{I}}+J_{\mathrm{II}}=\frac{L^{2}}{2 \alpha^{\prime}}=J .
$$


We stress that the outgoing strings represent excited string states which do not have maximum angular momentum.

For completeness, we also give the results in the case of open strings. The solutions are simply obtained by the formal substitution $2 \sigma \rightarrow \sigma, 2 \tau \rightarrow \tau$ in eqs. (2.1), (2.16) - (2.19), with the new $\sigma$ defined in the intervals $0<\sigma<\pi a$ and $0<\sigma<\pi(1-a)$, respectively. The expressions for $E_{I}, p_{I}^{y}, J_{I}, l_{I}, S_{I}$ are the same as above with an extra factor $1 / 2$, and similarly for the string II.

\subsection{Closed formulas for the outgoing string solutions}

We can describe the classical closed-string dynamics by means of left and right motion, in terms of the coordinates $\sigma^{ \pm}=\sigma \pm \tau$ :

$$
X^{\mu}(\sigma, \tau)=X_{+}^{\mu}\left(\sigma^{+}\right)+X_{-}^{\mu}\left(\sigma^{-}\right) .
$$

The constraint is $\eta_{\mu \nu} \partial_{ \pm} X_{ \pm}^{\mu} \partial_{ \pm} X_{ \pm}^{\nu}=0$.

The initial string (2.1) is described by:

$$
X_{ \pm}\left(\sigma^{ \pm}\right)=\frac{L}{2} \cos \left(2 \sigma^{ \pm}\right), \quad Y_{ \pm}\left(\sigma^{ \pm}\right)= \pm \frac{L}{2} \sin \left(2 \sigma^{ \pm}\right), \quad X_{ \pm}^{0}\left(\sigma^{ \pm}\right)= \pm L \sigma^{ \pm} .
$$

As before, $\sigma \in[0, \pi)$.

At $\tau=0$ the string splits into two pieces: $X_{ \pm}^{\mu}\left(\sigma^{ \pm}\right) \rightarrow\left\{X_{\mathrm{I} \pm}^{\mu}\left(\sigma^{ \pm}\right), X_{\mathrm{II} \pm}^{\mu}\left(\sigma^{ \pm}\right)\right\}$. The two pieces are folded like the initial string and the splitting occurs at $\sigma=\frac{a \pi}{2}$ in the upper segment and at $\sigma=\pi-\frac{a \pi}{2}$ in the lower segment, with

$0<a<1$. The two resulting strings are determined as in the previous subsection by requiring continuity of the string coordinates and their first derivatives in $\tau$ at $\tau=0$, and by requiring periodicity in $\sigma: X_{\mathrm{I}}^{\mu}$ with period $\Delta \sigma=a \pi$ and $X_{\text {II }}^{\mu}$ with period $\Delta \sigma=(1-a) \pi$.

In the interval of $\sigma$ corresponding to string I (see eq. (2.4) ), the initial conditions at $\tau=0$ imply that $\partial_{\sigma} X(\sigma, 0)=X_{+}^{\prime}(\sigma)+X_{-}^{\prime}(\sigma)=X_{\mathrm{I}+}^{\prime}(\sigma)+X_{\mathrm{I}-}^{\prime}(\sigma)$ and $\partial_{\tau} X(\sigma, 0)=X_{+}^{\prime}(\sigma)+X_{-}^{\prime}(\sigma)=X_{+}^{\prime}(\sigma)-X_{-}^{\prime}(\sigma)$, which in turn imply that $X_{I+}(\sigma)=X_{+}(\sigma)$ and $X_{\mathrm{I}-}(\sigma)=X_{-}(\sigma)$ in this interval, and outside of the interval they are defined by the new periodic boundary condition $\sigma \rightarrow \sigma+a \pi$. This determines $X_{I}(\sigma, \tau)=X_{I+}\left(\sigma^{+}\right)+X_{I-}\left(\sigma^{-}\right)$in closed form. Similarly for the coordinate $Y$, and for the string II. 
The resulting expressions for $Y_{\mathrm{I}, \mathrm{II}}$ are the sum of two terms. One term corresponds to the momentum carried by the string: $\pm L \frac{2 \sin (a \pi)}{\pi} \tau$. The other term is periodic in $\sigma$ and its derivative in $\sigma$ has zero average.

It is convenient to rescale the world-sheet parameters of the resulting strings $\sigma^{ \pm} \rightarrow a \sigma^{ \pm}$for the string I, and $\sigma^{ \pm} \rightarrow(1-a) \sigma^{ \pm}$for the string II, in such a way that the period is $\Delta \sigma=\pi$ for both.

We get for the string I:

$$
\begin{aligned}
& X_{\mathrm{I} \pm}^{0}\left(\sigma^{ \pm}\right)= \pm L a \sigma^{ \pm} \\
& X_{\mathrm{I} \pm}\left(\sigma^{ \pm}\right)=\frac{L}{2} C_{\mathrm{I}}\left(\sigma^{ \pm}\right), Y_{\mathrm{I} \pm}\left(\sigma^{ \pm}\right)= \pm \frac{L}{2}\left[\frac{2 \sin (a \pi)}{\pi} \sigma^{ \pm}+S_{\mathrm{I}}\left(\sigma^{ \pm}\right)\right]
\end{aligned}
$$

where

$$
\begin{aligned}
& C_{\mathrm{I}}(\sigma)=\cos (2 a \sigma), \quad S_{\mathrm{I}}(\sigma)=\sin (2 a \sigma)-\frac{2 \sin (a \pi)}{\pi} \sigma \\
& \text { for } \quad 0 \leq \sigma<\frac{\pi}{2}, \\
& C_{\mathrm{I}}(\sigma)=\cos (2 a \sigma-a 2 \pi), \quad S_{\mathrm{I}}(\sigma)=\sin (2 a \sigma-a 2 \pi)-\frac{2 \sin (a \pi)}{\pi}(\sigma-\pi) \\
& \text { for } \quad \frac{\pi}{2} \leq \sigma<\pi .
\end{aligned}
$$

Similarly for the string II we get:

$$
\begin{aligned}
& X_{\mathrm{II} \pm}^{0}\left(\sigma^{ \pm}\right)= \pm L(1-a) \sigma^{ \pm} \\
& X_{\mathrm{II} \pm}\left(\sigma^{ \pm}\right)=\frac{L}{2} C_{\mathrm{II}}\left(\sigma^{ \pm}\right) \\
& Y_{\mathrm{II} \pm}\left(\sigma^{ \pm}\right)= \pm \frac{L}{2}\left[-\frac{2 \sin (a \pi)}{\pi} \sigma^{ \pm}+S_{\mathrm{II}}\left(\sigma^{ \pm}\right)\right]
\end{aligned}
$$

where

$$
\begin{aligned}
& C_{\mathrm{II}}(\sigma)=\cos (2(1-a) \sigma+a \pi), \\
& S_{\mathrm{II}}(\sigma)=\sin (2(1-a) \sigma+a \pi)+\frac{2 \sin (a \pi)}{\pi} \sigma \\
& \text { for } 0 \leq \sigma<\pi .
\end{aligned}
$$

These definitions are extended to any $\sigma$ by declaring that

$$
C_{\mathrm{I}, \mathrm{II}}(\sigma+\pi)=C_{\mathrm{I}, \mathrm{II}}(\sigma), \quad S_{\mathrm{I}, \mathrm{II}}(\sigma+\pi)=S_{\mathrm{I}, \mathrm{II}}(\sigma) .
$$


They solutions are equivalent to the solutions given by the Fourier expansion in the previous section.

The derivative in $\sigma$ of both $X_{\mathrm{I}}, Y_{\mathrm{I}}$ and $X_{\mathrm{II}}, Y_{\mathrm{II}}$ has a discontinuity at $\sigma^{ \pm}=\pi / 2$ and $\sigma^{ \pm}=0$ respectively. This discontinuity will appear as an angular bending in the (folded) shape of the strings I and II. Since $\sigma^{ \pm}=\sigma \pm \tau$, this angular bending will move along the string, as a function of $\tau$. We will return to this point in the last section.

\section{Comparison with the superstring quantum calculation}

The decay process described above by semiclassical splitting predicts that masses will be related by the following formulas:

$$
\begin{gathered}
M_{\mathrm{I}}=M_{\mathrm{I}}(a)=\frac{L}{\alpha^{\prime}} \sqrt{a^{2}-\frac{\sin ^{2}(\pi a)}{\pi^{2}}}, \\
M_{\mathrm{II}}=M_{\mathrm{II}}(a)=\frac{L}{\alpha^{\prime}} \sqrt{(1-a)^{2}-\frac{\sin ^{2}(\pi a)}{\pi^{2}}} .
\end{gathered}
$$

These relations define a function $M_{\mathrm{I}}=M_{\mathrm{I}}\left(M_{\mathrm{II}}\right)$.

In ref. [1], the full quantum calculation of the decay was done in the ten dimensional type II superstring theory. The decay rate is obtained by extracting the imaginary part of the genus one self-energy of the massive particle: $\Gamma=\operatorname{Im} \frac{\Delta M^{2}}{2 M}$.

This calculation is complicated: it combines expansions of theta functions and resummations, saddle-point evaluation of some integrals. Nevertheless, it is an exact genus one result in the large $M$ limit, since the only approximation involved are saddle-point approximations, which become exact as $\alpha^{\prime} M^{2} \gg 1$.

Here we explain briefly the idea of the method and the result. We compute the contribution to $\operatorname{Im}\left(\Delta M^{2}\right)$ of the decay channel corresponding to the

states with masses $M_{1}, M_{2}$ : let us call $\frac{d^{2} \operatorname{Im}\left(\Delta M^{2}\right)}{d M_{1}^{2} d M_{2}^{2}}$ that contribution per unit $d M_{1}^{2} d M_{2}^{2}$. The one-loop self-energy, derived first in [9], is represented as an integral of some combination of theta functions, the integration being over the torus complex modulus and over a complex (vertex position) coordinate on the torus surface. The integration is formally divergent, and it is computed by a standard analytic continuation. 
A key point is to write the integrand as a sum of holomorphically factorized quantities. The selection of a particular decay channel corresponds to a particular term in the Taylor expansion of the holomorphic factors, as it is recognized by comparison with field theory Feynman diagrams. Cauchy contour integrals and saddle point techniques are used for getting the holomorphic expansion, similarly to the well known procedure for computing the entropy of a state in string theory. In this way we obtained:

$$
\frac{d^{2} \operatorname{Im}\left(\Delta M^{2}\right)}{d M_{1}^{2} d M_{2}^{2}} \sim g_{s}^{2} M^{-3} \exp \left[2 M^{2} S_{0}\left(M_{1} / M, M_{2} / M\right)\right],
$$

with $S_{0} \leq 0$ (given in fig. 1 of [11]).

Therefore in the large mass limit the dominant decay channel corresponds to values of $M_{1,2}$ for which $S_{0}=0$, i.e. the masses of the decay products are correlated, modulo processes which are exponentially suppressed. This effect may come as a surprise, since one might have expected a sizable string vertex coupling three string states of arbitrary masses $M, M_{1}, M_{2}$.

Figure 1 shows the relation $M_{\mathrm{I}}=M_{\mathrm{I}}^{\text {quantum }}\left(M_{\mathrm{II}}\right)$, corresponding to $S_{0}=0$, found numerically in [11] (and shown there in fig. 3). . In the same figure, we have superposed the analytic function $M_{\mathrm{I}}=M_{\mathrm{I}}\left(M_{\mathrm{II}}\right)$ defined by (3.1), (3.2). We see that they coincide, the analytic semiclassical curve fully matches the curve of [11] obtained by a one-loop calculation.

This precise match is also surprising. Although one expects that for a large mass the initial highly excited quantum string state is well described by a classical solution, it is not a priori obvious that the precise relation between masses should be implied by a classical spontaneous splitting process. Moreover, the decay products are not states of maximum angular momentum and it is not obvious that a semiclassical description would be applicable for them. The accurate coincidence of the two curves in figure 1 also confirms the results of [11].

Further, in the calculation of [11], it is also possible to isolate the contribution of a given orbital angular momentum $l_{0}$. In fact, by comparison with the field theory expressions for one-loop Feynman diagrams, one learns that the sum over the possible $l_{0}$ contributions corresponds to the sum over the holomorphically factorized terms (in eq. (5.4) of [11], a term with given $l_{0}$ has $l_{0}=2 N-\left(m_{1}+m_{2}\right)-2=$ fixed, $\left.N=J / 2\right)$.

However, $l_{0}$ is not a quantum number of the final states, and in fact the quantum computation of $\operatorname{Im}\left(\Delta M^{2}\right)$ is expressed as the modulus square of a 


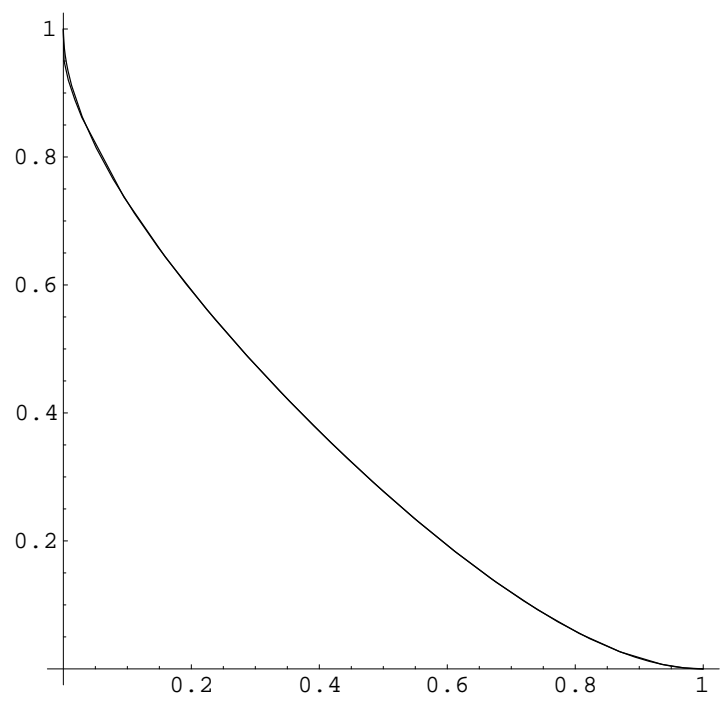

Figure 1: The curve $M_{\mathrm{I}}=M_{\mathrm{I}}\left(M_{\mathrm{II}}\right)$ defined by eqs. (3.1), (3.2). It is superposed with the similar curve obtained in [1] from the direct quantum one-loop calculation.

sum over amplitudes which are alternating in sign. Therefore, the classical value of $l_{0}$ does not correspond to a well defined mean value. In order to compare the quantum computation with the classical configuration of outgoing states $M_{1}, M_{2}$ with given $l_{0}$, we have taken as quantum value of $l_{0}$ the one for which the amplitude is maximal in absolute value. That maximal amplitude corresponds to a value of $l_{0}$ which depends on $M_{1}, M_{2}$. We take the relevant values for $M_{1,2}$ to be those along the curve of Figure 1, and then compare this result to $l_{\mathrm{I}}(a)+l_{\mathrm{II}}(a)$ computed semiclassically (see eqs. (2.13), (2.21) ). This comparison is shown in Figure 2.

We see that the two curves are quite close. We think that the small discrepancy is due to the fact that there is not a well defined quantum value of $l_{0}$. It is still remarkable that the semiclassical and quantum calculation give so close results, with the correct normalization which comes automatically without any tuning. 


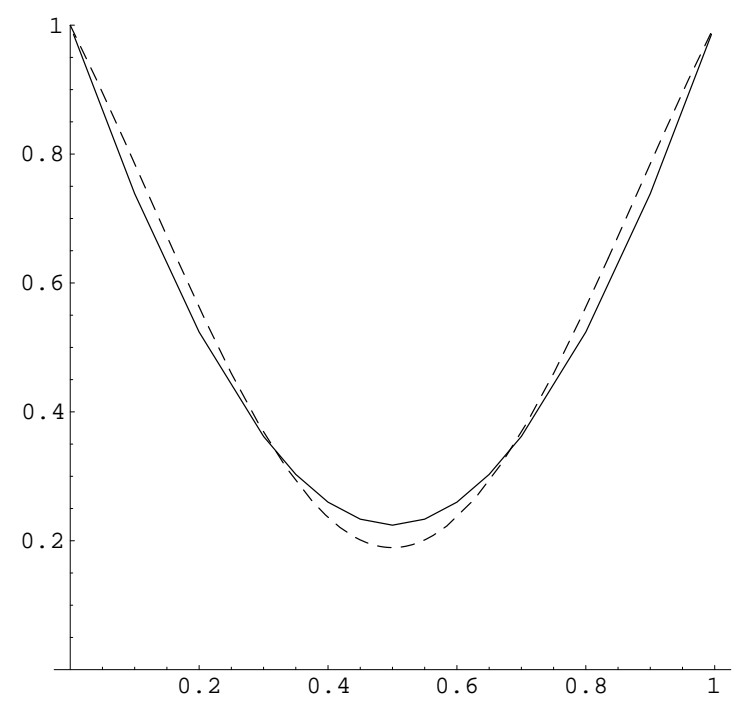

Figure 2: Plot of the classical (dashed line) and quantum (solid line) value for $\left(1-l_{0} / J\right)$ $\left(l_{0}=\right.$ orbital angular momentum) as a function of the parameter $a$ (which defines the breaking point of the string).

\section{Decay rate and description of the motion}

For quantum states with large occupation numbers admitting a semiclassical description, one expects for the lifetime an expression of the form

$$
\mathcal{T}=\Gamma^{-1}=\text { const. } \frac{1}{g_{s}^{2}} \mathcal{T}_{0} \exp \left[-2 \frac{L^{2}}{\alpha^{\prime}} S_{0}^{M a x}\right]
$$

This is precisely the form of the result (3.3) that arises from the explicit quantum calculation. We have seen that in general $S_{0} \leq 0$ and that the maximum of $S_{0}$ corresponds to the classical solution for which $S_{0}^{M a x}=0$. The total contribution to $\Gamma$ is obtained by performing the integral over $d M_{1}^{2}, d M_{2}^{2}$ of (3.3) [11. Only a small neighborhood around the curve of fig. 1 contributes, since other regions are suppressed exponentially. Using the semiclassical form (3.3) of $S_{0}$ and expanding in the vicinity of the curve, one is left with a Gaussian integral in the orthogonal direction of the curve which produces an additional factor of $1 / \sqrt{N}$. This procedure gives (4.1) with $\mathcal{T}_{0} \cong L$, i.e. $\mathcal{T}=\Gamma^{-1}=$ const. $\frac{\alpha^{\prime}}{g_{s}^{2}} M$, which was the result reported in [11]. However, while the saddle point approximation gives an accurate formula for the exponential part of the decay rate, obtaining the power behavior is subtle in the present case, due to the vanishing of some determinants in the vicinity 
of the curve of fig. 1. The correct power behavior is being considered in a work in progress.

Having the exact solutions for the outgoing strings I and II, it is interesting to describe the main features of their motion. Figures 3 and 4 are plots of a sequence of pictures of the string I and II after the splitting, for different breaking points: $a=0.4, a=0.15$ respectively. We see that the outgoing closed string remain folded, exhibiting a rotating motion. The figures 3 and 4 were made using the Fourier series formulas of section 2.1. One can check that the same plot is obtained using the formulas of section 2.2. Figures 5,6,7 are a plot of the world-sheet for the breaking point at $a=0.4$.

The most salient feature that can be observed from the figure is that the breaking of the strings creates an angular bending, or kink, which then travels back and forth all along each string. Remarkably, each string is straight except at the bending point. One might wonder whether this feature is generic, at least for the breaking of an open string: the kink is produced by the jump of the first derivative at the splitting point; it is locally created, thus its occurrence should not depend on whether the string has maximum angular momentum (i.e. whether it is straight or curved).

Another important feature is that the angles of the bendings of the strings I and II sum up $180^{\circ}$, and they are given by

$$
\theta_{\mathrm{I}}=a \pi, \quad \theta_{\mathrm{II}}=(1-a) \pi .
$$

Note that they are in relation with the energies of the strings. When $a=1 / 2$ both angles are $\pi / 2$.

The formulas (4.2) can be proved by using the solutions of section 2.2. Consider the string I at a given instant $\tau$. The derivative $\frac{d Y_{\mathrm{I}}}{d X_{\mathrm{I}}}$ has the same discontinuity in both upper and lower segments of the closed string. In one segment the discontinuity originates from the discontinuity in $\partial_{\sigma} X_{\mathrm{I}+}$ at $\sigma_{1}=\frac{\pi}{2}-\tau$. In the other segment, it originates from the discontinuity of $\partial_{\sigma} X_{\mathrm{I}-}$ at $\sigma_{2}=\frac{\pi}{2}+\tau$.

For $a<1 / 2$, the bending angle $\theta_{I}$ of the string $\mathrm{I}$ is an acute angle. It can be computed in particular at $\sigma_{1}$, where it is given by

$$
\theta_{\mathrm{I}}=\left.\arctan \frac{d Y_{\mathrm{I}}}{d X_{\mathrm{I}}}\right|_{\sigma_{1}-\epsilon}-\left.\arctan \frac{d Y_{\mathrm{I}}}{d X_{\mathrm{I}}}\right|_{\sigma_{1}+\epsilon} .
$$

Using the explicit form of the solutions, we obtain

$$
\left.\frac{d Y_{\mathrm{I}}}{d X_{\mathrm{I}}}\right|_{\sigma_{1}-\epsilon}=\left.\frac{\partial_{\sigma} Y}{\partial_{\sigma} X}\right|_{\sigma_{1}-\epsilon}=\tan (2 a \tau),
$$




$$
\left.\frac{d Y_{\mathrm{I}}}{d X_{\mathrm{I}}}\right|_{\sigma_{1}+\epsilon}=\tan (2 a \tau-a \pi)
$$

Thus

$$
\theta_{\mathrm{I}}=2 a \tau-(2 a \tau-a \pi)=a \pi .
$$

Similarly, one finds $\theta_{\mathrm{II}}$ as in (4.2).

\section{Acknowledgements:}

We thank J. Maldacena for suggesting the basic idea that led to this investigation, namely that the relation between the masses $M_{1}, M_{2}$ of the decay products found in [11] could be explained in terms of spontaneous splitting of the rotating string solution. R.I. acknowledges partial support by the EEC grant HPRN-CT-2000-00131.

\section{References}

[1] M. B. Green and G. Veneziano, Phys. Lett. B 36, 477 (1971).

[2] D. Mitchell, N. Turok, R. Wilkinson and P. Jetzer, Nucl. Phys. B 315, 1 (1989) [Erratum-ibid. B 322, 628 (1989)].

[3] J. Dai and J. Polchinski, Phys. Lett. B 220, 387 (1989).

[4] H. Okada and A. Tsuchiya, Phys. Lett. B 232, 91 (1989).

[5] B. Sundborg, Nucl. Phys. B 319, 415 (1989).

[6] R. B. Wilkinson, N. Turok and D. Mitchell, Nucl. Phys. B 332, 131 (1990).

[7] D. Mitchell, B. Sundborg and N. Turok, Nucl. Phys. B 335, 621 (1990).

[8] D. Amati and J. G. Russo, Phys. Lett. B 454, 207 (1999) arXiv:hep-th/9901092.

[9] R. Iengo and J. Kalkkinen, JHEP 0011, $025 \quad$ (2000) arXiv:hep-th/0008060.

[10] J. L. Manes, Nucl. Phys. B 621, 37 (2002) arXiv:hep-th/0109196. 
[11] R. Iengo and J. G. Russo, JHEP 0211, 045 (2002) arXiv:hep-th/0210245.

[12] H. J. de Vega, J. Ramirez Mittelbrunn, M. Ramon Medrano and N. Sanchez, Phys. Rev. D 52, 4609 (1995) arXiv:hep-th/9502049.

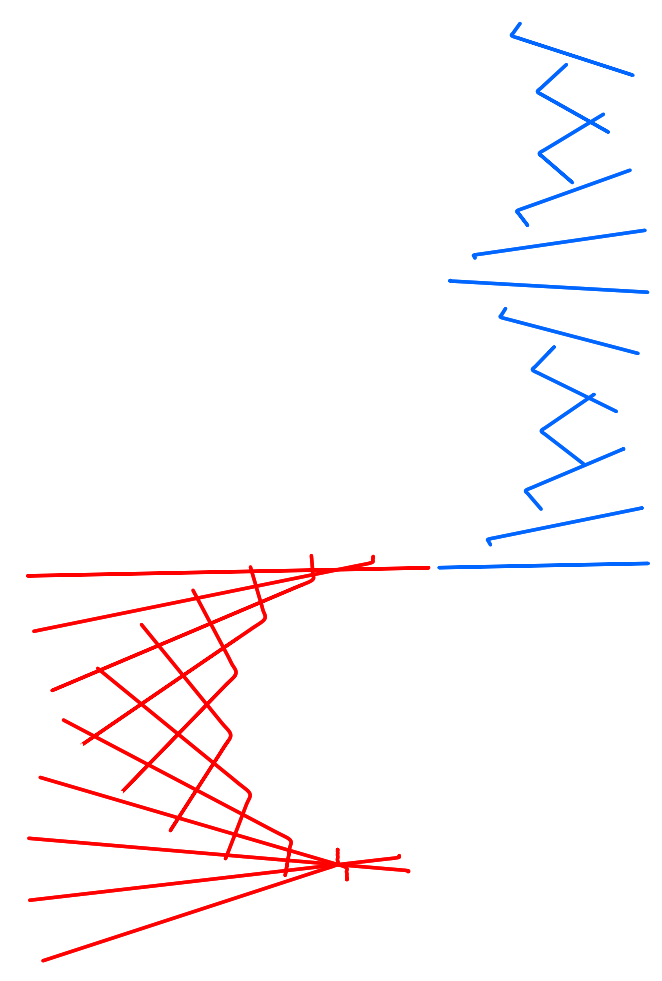

Figure 3: Sequences of pictures of the closed string after the splitting for $a=0.4$. 


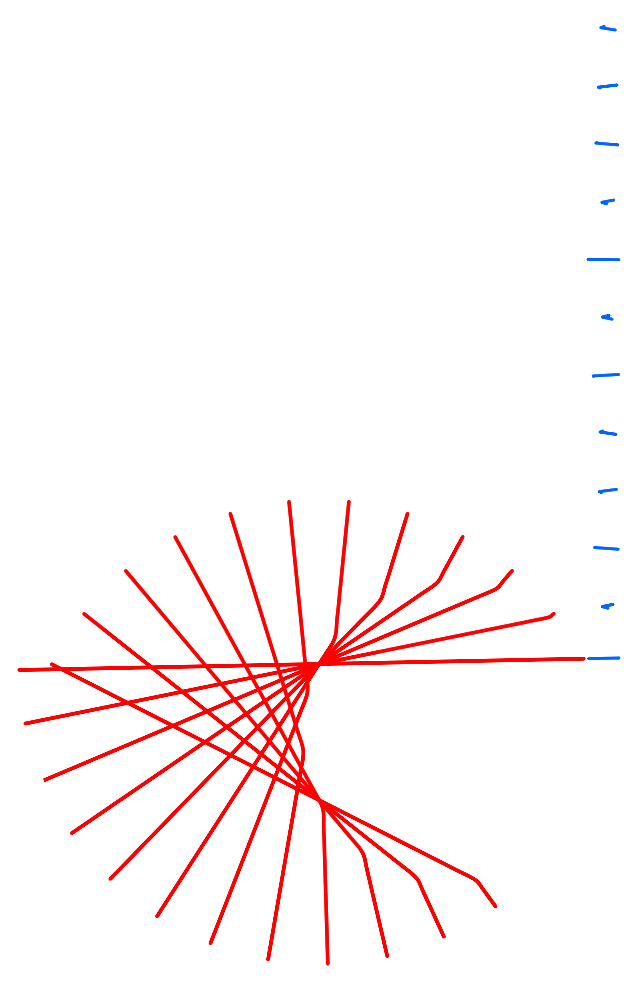

Figure 4: Sequences of pictures of the closed string after the splitting for $a=0.15$. One can see the slow translational motion of the large string, which is a small deformation of the original rotating string solution, with a bending angle $0.85 \pi$. The small string moves very fast and the bending angle is acute, equal to $0.15 \pi$. 


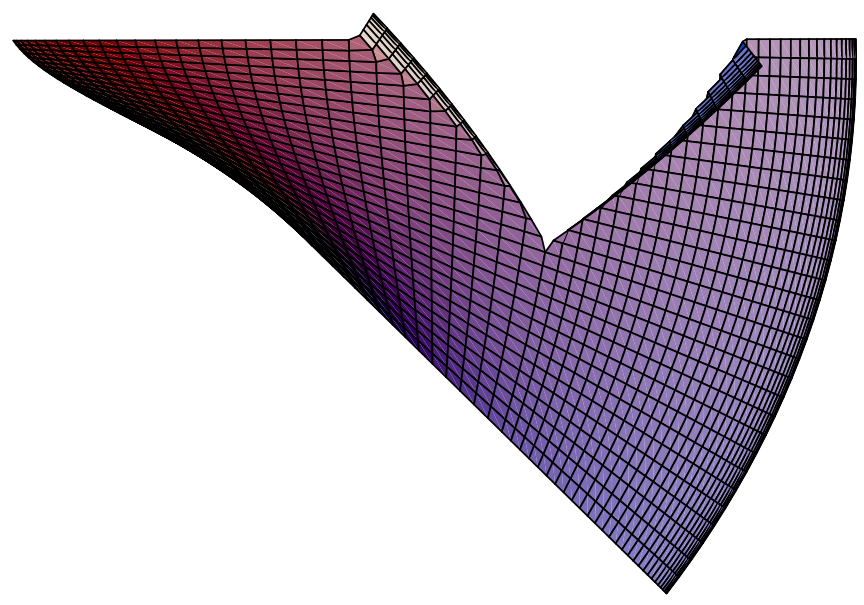

Figure 5: Surface swept by the strings during the splitting process (lateral view) . 


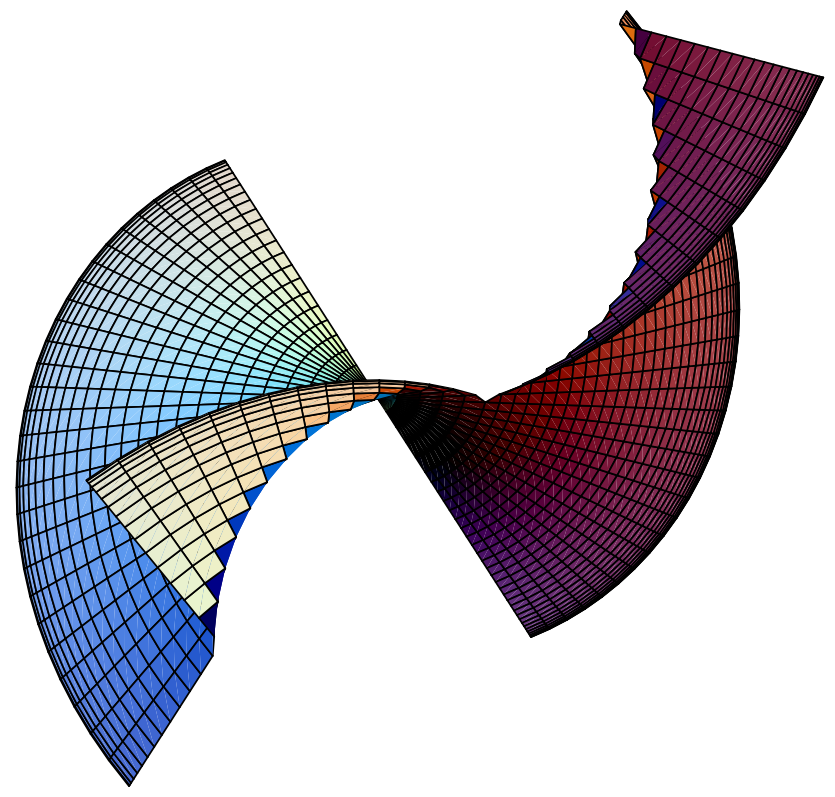

Figure 6: Surface swept by the strings during the splitting process (view from the top, larger time interval) . 


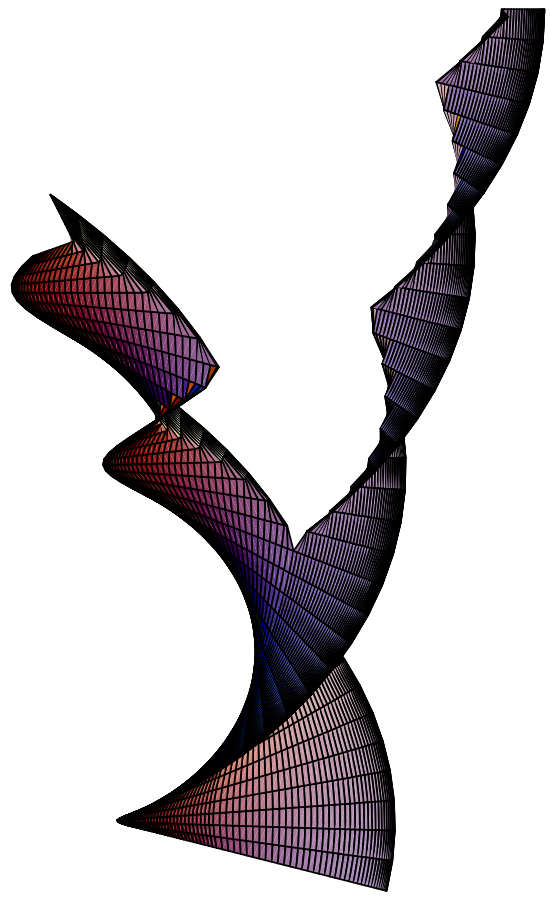

Figure 7: Surface swept by the strings during the splitting process (lateral view, even larger time interval) . 